\title{
ANALYTICAL MODEL OF TRANSLATIONAL NONEQUILIBRIUM FLOW WITH INTERNAL DEGREES OF FREEDOM
}

\author{
I. I. Lipatov ${ }^{1}$, M. M. Kuznetsov ${ }^{2}$, and L. V. Smotrova ${ }^{2}$ \\ ${ }^{1}$ Central Aerohydrodynamic Institute (TsAGI) \\ 1 Zhukovsky Str., Zhukovsky, Moscow Region 140180, Russia \\ ${ }^{2}$ Moscow State Regional University \\ 10a Radio Str., Moscow, Russia
}

\begin{abstract}
On the basis of the kinetic equation for gases with internal degrees of freedom, an asymptotical analysis of hypersonic rarefied gas flows is conducted. Nonlinear transfer equations describing a hypersonic flow near a blunt body are derived. The results of computations are compared with those obtained by solving the viscous shock layer equations along the with Boltzmann equations.
\end{abstract}

\section{INTRODUCTION}

One of the important problems of the kinetic theory of gases is the problem of determining the boundaries of applicability of continuum mechanics for hypersonic flows in the transitional regime. Despite a large amount of theoretical, computational, and experimental papers, there are still no publications dealing with criteria of violation of the continuum description of hypersonic flows based on the Navier-Stokes equations or application of their asymptotic models. Such a problem can be solved only on the basis of the molecular-kinetic approach.

\section{EQUATIONS}

The thin viscous shock layer approach (Chang's model [1]) is widely used to describe viscous heat-conducting hypersonic gas flows. In [2], the asymptotic Navier-Stokes equations were analyzed for the case of an axisymmetrical hypersonic flow of a perfect gas near blunted bodies under the conditions

$$
\mathrm{M}_{\infty} \rightarrow \infty ; \quad \varepsilon=\frac{R_{g} T_{0}}{V_{\infty}^{2}} \sim \frac{\rho_{\infty}}{\rho_{*}} \rightarrow 0 ; \quad \operatorname{Re}_{0}=\frac{\rho_{\infty} V_{\infty} R_{0}}{\mu_{0}} \rightarrow \infty
$$

This is an Open Access article distributed under the terms of the Creative Commons Attribution License 4.0, which permits unrestricted use, distribution, and reproduction in any medium, provided the original work is properly cited. 
where $\mathrm{M}_{\infty}, V_{\infty}$, and $\rho_{\infty}$ are the Mach number, the velocity, and the density in the external flow, respectively; $T_{0}=\varepsilon V_{\infty}^{2} / R_{g}$ is the stagnation temperature of the external flow ( $R_{g}$ is the universal gas constant); $\mu_{0}=\mu\left(T_{0}\right)$ is the viscosity coefficient determined at the stagnation temperature; $\rho_{*}$ is the typical density value in the shock layer; $R_{0}$ is the blunted body radius at the stagnation point; and $\operatorname{Re}_{0}$ is the Reynolds number.

As a result of the analysis, the following flow regimes in the shock layer, which are characterized by the parameters $K=\left(\varepsilon^{5 / 2} \operatorname{Re}_{0}\right)^{-1}$ and $N=\left(\varepsilon \operatorname{Re}_{0}\right)^{-1}$, were determined:

$-K \ll 1$ boundary layer flow regime;

- $K \sim 1$ vortical interaction regime; and

$-N \sim 1$ viscous shock layer regime, where viscous effects are important in the entire disturbed flow region.

Equations uniformly valid in the whole shock layer for the cases analyzed coincide with the equations deduced in [1] within the framework of the so-called two-layer model.

An asymptotic analysis was performed for a polyatomic gas flow in a thin shock layer on the basis of kinetic equation for gases with internal degrees of freedom in $[3,4]$.

As a result of the limiting procedure (1) for the case of $N \sim 1$ in the infinite chain of distribution function moments derived from the kinetic equation, the following closed system of microscopic equations was obtained to describe the polyatomic gas flow in the shock layer.

In a curvilinear coordinate system fitted to with the body surface, the conservations equations for an axysimmetric $(\nu=1)$ or planar $(\nu=0)$ gas flow in the viscous shock layer have the following form $[3,4]$ :

$$
\left.\begin{array}{r}
\frac{\partial}{\partial x}\left(r^{v} \rho u\right)+\frac{\partial}{\partial y}\left(r^{v} \rho v\right)=0 ; \\
\rho\left(\frac{u}{h_{1}} \frac{\partial u}{\partial x}+v \frac{\partial u}{\partial y}+\varepsilon \frac{u v}{R h_{1}}\right)+\frac{\varepsilon}{h_{1}} \frac{\partial P_{x x}}{\partial x}+\frac{\partial P_{x y}}{\partial y}=0 ; \\
\rho\left[\varepsilon\left(\frac{u}{h_{1}} \frac{\partial v}{\partial x}+v \frac{\partial v}{\partial y}\right)-\frac{u^{2}}{R h_{1}}\right]+\frac{\partial P_{y y}}{\partial y}=0 ; \\
\rho\left(\frac{u}{h_{1}} \frac{\partial H}{\partial x}+v \frac{\partial H}{\partial y}\right)+\frac{\partial}{\partial y}\left(q_{y}+2 u P_{x y}\right)=0 .
\end{array}\right\}
$$

Here, the closing relations have the form:

$$
\left.\begin{array}{c}
p=\rho T_{t} ; \quad T_{t}=T+\Delta T ; \quad H=h+u^{2}+v^{2} ; \\
h=T+2 \varepsilon \Delta T ; \quad T=\frac{1}{c_{v}}\left(\frac{3}{2} R_{g} T_{t}+c_{\mathrm{in}} T_{\mathrm{in}}\right) ; \quad c_{v}=\frac{3}{2} R_{g}+c_{\mathrm{in}} ;
\end{array}\right\}
$$




$$
\left.\begin{array}{c}
P_{x x}=p \frac{1+2 \Omega^{2}}{1+(2 / 3) \Omega^{2}} ; \quad P_{y y}=\frac{p}{1+(2 / 3) \Omega^{2}} ; \quad P_{x y}=-N \frac{P_{y y}}{p} \mu \frac{\partial u}{\partial y} \\
q_{y}=-N \operatorname{Pr}^{-1} \frac{P_{y y}}{p} \mu \frac{\partial h}{\partial y} ; \quad \Omega=N \frac{\mu}{p} \frac{\partial u}{\partial y}=-\frac{P_{x y}}{P_{y y}} \\
\frac{\Delta T}{T_{t}}=\frac{c_{\text {in }}}{c_{v}} \frac{T_{t}-T_{\text {in }}}{T_{t}}=\frac{2}{3} \frac{c_{\text {in }}}{c_{v}} \frac{p}{\mu} \tau_{\text {in }} \frac{\Omega^{2}}{1+(2 / 3) \Omega^{2}}
\end{array}\right\}
$$

In (2) and closing relations (3) and (4), $x$ is the distance calculated along the body surface from the stagnation point; $y$ is the distance calculated in the normal direction from the surface; $u$ and $v$ are the velocity components in the $x$ and $y$ directions; $H$ is the total enthalpy; $\varepsilon=(\gamma-1) /(2 \gamma)$ ( $\gamma$ is the ratio of specific heats); $R(x)$ is the curvature radius of the body; $h_{1}$ is the Lame coefficient; $r$ is the distance from the point of space to the body axis; $T_{t}$ and $T_{\text {in }}$ are the temperatures of the translational and internal degrees of freedom; $c_{\text {in }}$ is the heat capacity of the internal molecular degree of freedom; $\mu$ is the viscosity; Pr is the Prandtl number; and $\tau_{\text {in }}$ is the time of relaxation of the energy of internal degrees of freedom of molecules. It is assumed that energy exchange occurs between the translational and internal degrees of freedom; so, the parameter $\alpha \sim \tau_{\mathrm{el}} / \tau_{\text {in }} \sim 1$ $\left(\tau_{\mathrm{el}} \sim \mu / p\right.$ is the time of relaxation of the translational energy of the degrees of freedom of molecules).

In Eqs. (1) and (2), the density $\rho$ is divided by $\varepsilon^{-1} \rho_{\infty}, u-$ by $V_{\infty}, v-$ by $\varepsilon V_{\infty}$, the stress tensor components $P_{x x}, P_{y y}$, and $P_{x y}$ and the pressure $p-$ by $\rho_{\infty} V_{\infty}^{2}, H-$ by $V_{\infty}^{2} / 2$, the normal component of the heat flux vector $q_{y}-$ by $\rho_{\infty} V_{\infty}^{3} / 2$, the temperatures $T, T_{t}$, and $T_{\text {in }}-$ by $T_{0}$, the viscosity $\mu-$ by $\mu_{0}$, the distances $x, r$, and $R$ - by $R_{0}$, the distance $y$ - by $\varepsilon R_{0}$, and $\tau_{\text {in }}-$ by $\mu_{0} /\left(\rho_{\infty} V_{\infty}^{2}\right)$.

In contrast to the thin shock layer approximation, some terms containing $\varepsilon$ are retained in Eqs. (2), because Chang's model is invalid in the region of shock layer separation near the point of zero pressure on the surface; so, the computation downstream from this point is impossible.

Equations (2)-(4) are characterized by nonlinear stress tensor components along with the heat flux vector on the function $\Omega=N(\mu(\partial u / \partial y) / p)$. The system of Eqs. (2)-(4) will be defined as nonlinear transfer equations.

It was shown [3] that the boundary conditions on the shock wave at $y=y_{s}$ for Eqs. (2) are the relations analogous to the generalized Rankine-Hugoniot relations:

$$
\begin{aligned}
v_{s} & =u_{s} \frac{d y_{s}}{d x}-\varepsilon \rho_{s}^{-1} \frac{\sin \beta}{\cos \beta_{s}} \\
u_{s} & =\cos \beta \cos \beta_{s}+\varepsilon \rho_{s}^{-1} \sin \beta \sin \beta_{s}(\sin \beta)^{-1}\left(P_{x y}\right)_{s} \\
H_{s} & =1+(\sin \beta)^{-1}\left(q_{y}+2 u P_{x y}\right)_{s} \\
\left(P_{y y}\right)_{s} & =\left(1-\varepsilon \rho_{s}^{-1}\right) \sin ^{2} \beta
\end{aligned}
$$


Here, $\beta_{s}$ is the angle between the shock wave and the body; $\beta$ is the angle between the shock wave and the axis of symmetry of the body; $P_{x y}, P_{y y}$, and $q_{y}$ are determined by relations (3) and (4).

Owing to estimates obtained in [3], the slip velocity and temperature jump on the body surface are of the order of $N \sqrt{\varepsilon g_{w}}\left(g_{w}=T_{w} / T_{0}, T_{w}\right.$ is the surface temperature). At $N \sim 1$ and $\varepsilon \ll 1, g_{w} \leq 1$, these effects are negligible. Then, the following condition is valid for an impermeable surface at $y=0$ :

$$
u=v=0 ; \quad T=T_{w} .
$$

For $N \ll 1$, relations (3) and (4) can be expanded into series containing degrees of $N$ :

$$
\begin{aligned}
P_{x x} & =p\left[1+\frac{4}{3} N^{2}\left(\frac{\mu}{p} \frac{\partial u}{\partial y}\right)^{2}+O\left(N^{4}\right)\right] ; \\
P_{y y} & =p\left[1-\frac{2}{3} N^{2}\left(\frac{\mu}{p} \frac{\partial u}{\partial y}\right)^{2}+O\left(N^{4}\right)\right] \\
P_{x y} & =-N \mu \frac{\partial u}{\partial y}\left[1-\frac{2}{3} N^{2}\left(\frac{\mu}{p} \frac{\partial u}{\partial y}\right)^{2}+O\left(N^{4}\right)\right] ; \\
q_{y} & =-N \operatorname{Pr}^{-1} \mu \frac{\partial h}{\partial y}\left[1-\frac{2}{3} N^{2}\left(\frac{\mu}{p} \frac{\partial u}{\partial y}\right)^{2}+O\left(N^{4}\right)\right] ; \\
\frac{\Delta T}{T_{t}} & =\frac{2}{3} \frac{c_{\text {in }}}{c_{v}} \frac{p}{\mu}\left[N^{2}\left(\frac{\mu}{p} \frac{\partial u}{\partial y}\right)^{2}+O\left(N^{4}\right)\right] .
\end{aligned}
$$

Terms containing $N, N^{2}$, and $N^{3}$ in relations (5) are also contained in the Navier-Stokes, Burnett, and super-Burnett approximations of the stress tensor and heat flux [5]. It may be concluded that there may be a significant difference between the translational and internal degrees of freedom at $N \sim 1$. If $N \ll 1$ in Eqs. (2)-(4), the terms containing $N^{2}$ and higher degrees of $N$ in the viscous shock layer equations may be deduced to equations that contain all terms of the Euler equations terms and of the boundary layer equations. Thus, the phenomenological approach based on limit (1) in the Navier-Stokes equations is valid only for $N \ll 1$. The viscous layer regime for which $N \sim 1$ is not described as the asymptotic approach of the Navier-Stokes equations and nonlinear transfer equations (2)-(4) should be used. In this work, a hypersonic flow of molecular nitrogen in the shock layer near a spherically blunted body is investigated numerically on the basis of the viscous shock layer equations along with the nonlinear transfer equations (2)-(4). The method of global iterations was used in the computations [6]. The computational results were compared with the results obtained in [7] by solving the kinetic Boltzman equation using the Monte-Carlo method. As in [7], it was assumed that the vibrational degrees of freedom are frozen. The time of relaxation of the energy of rotational degrees of freedom was determined on the basis of Parker's model [8]. 


\section{RESULTS}

The results of the computations showed that the rotational degrees of freedom are in essential nonequilibrium for relatively small Reynolds number values $\operatorname{Re}_{\infty} \sim 10^{2}\left(\operatorname{Re}_{\infty}=\rho_{\infty} V_{\infty} R_{0} / \mu_{\infty}\right.$ where $\mu_{\infty}$ is the viscosity at the external flow temperature), and the value of $\Delta T / T_{t}$ grows with the distance from the stagnation line. As a result, the translational temperature and shock layer thickness are greater than the corresponding values in the equilibrium regime.

As an example, Figs. 1-3 show the results of the computations for a hypersonic flow near spherical bluntness at $\mathrm{M}_{\infty}=26, \mathrm{Re}_{\infty}=65$, and $T_{w} / T_{0}=0.014$.

The distribution of the Stanton number St $=-q_{y} /\left(\rho_{\infty} V_{\infty}\left(H_{0}-H_{w}\right)\right)$ is depicted in Fig. 1 and the distribution of the skin friction coefficient $C_{f}=-2 P_{x y} /\left(\rho_{\infty} V_{\infty}^{2}\right)$ on the sphere surface is depicted in Fig. 2.

The results obtained in this work on the basis of the viscous shock layer equations (curves 1) were compared with the data obtained on the basis of the nonlinear transfer equations (2)-(4) (curves 2) and with the data obtained in [7] on the basis of solving the Boltzmann equation by the Monte-Carlo method (curves 3). It may be concluded that the results of the nonlinear transfer equations agree better with the Monte-Carlo predictions. At the same time, the viscous shock layer equations overpredict the heat flux and skin friction values on the bluntness surface. The maximum difference is reached in the mid-ship section and is of the order of $30 \%-40 \%$. The profiles of the dimensionless temperature $\bar{T}=T / T_{\infty}$ at $x / R_{0}=1.4$ (the symbols are the same as those in Figs. 1 and 2) are presented in Fig. 3.

It may be concluded that in comparison with the results obtained by the viscous shock layer equations, the results obtained on the basis of Eqs. (2)-(4) provide a more accurate description of the flow structure in the shock layer.

The numerical analysis conducted in [9] showed that the viscous shock layer equations with the velocity slip and temperature jump conditions provide

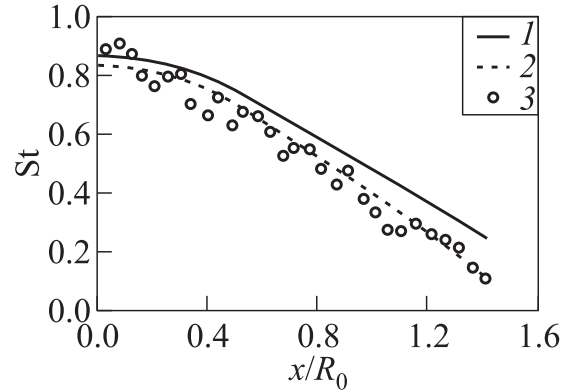

Figure 1 Distribution of the Stanton number St $=-q_{y} /\left(\rho_{\infty} V_{\infty}\left(H_{0}-H_{w}\right)\right)$

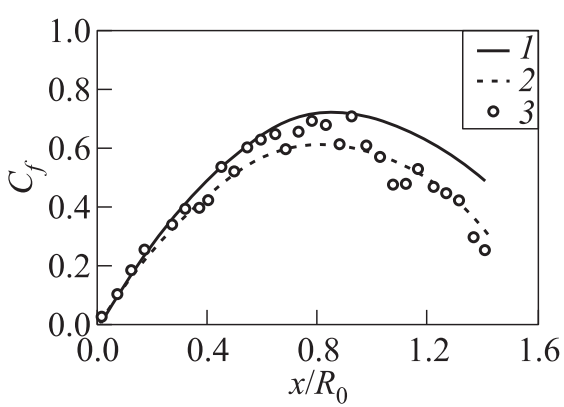

Figure 2 Distribution of the skin friction coefficient $C_{f}=-2 P_{x y} /\left(\rho_{\infty} V_{\infty}^{2}\right)$ on the sphere surface 


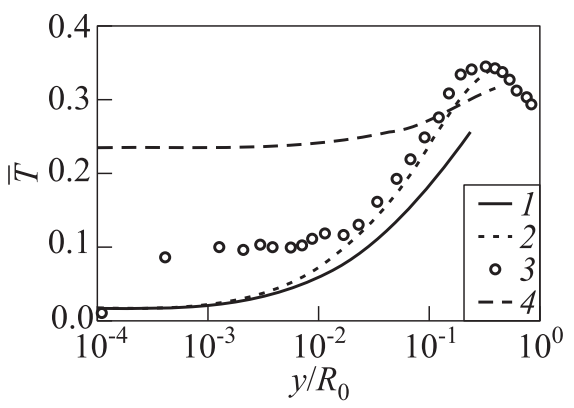

Figure 3 The profiles of the dimensionless temperature $\bar{T}=T / T_{\infty}$ at $x / R_{0}=1.4$ satisfactory data for relatively small Reynolds numbers. In this case, however, the velocity and temperature near the surface were significantly overpredicted (curve 4 in Fig. 3), as compared with the results obtained on the basis of the Boltzmann equation. It may be concluded that the nonlinear transfer equations provide a better description of the hypersonic flow near a blunted body than the viscous shock layer equations.

\section{ACKNOWLEDGMENTS}

The authors are grateful to the Russian Foundation for Basic Research (Grant 12-07-31172 young_a and Grants of the President of the Russian Federation (NSh-1495.2012.8 and MK-1969.2012.9) for the support of this study.

\section{REFERENCES}

1. Cheng, H.K. 1963. The blunt body problem in hypersonic flow at low Reynolds number. IAS Paper No.63-92. 100 p.

2. Bush, W. B. 1964. On the viscous hypersonic blunt body problem. J. Fluid Mech. 20(3):353-367.

3. Kuznetsov, M. M., and V.S. Nikol'sky. 1985. Hypersonic polyatomic viscous gas flow kinetic analysis in a thin 3-D shock layer. TsAGI Scientific Papers. 16(3):38-49.

4. Kuznetsov, M. M., and V.S. Nikol'sky. 1986. Kinetic theory of limiting hypersonic viscous gas flows. Numerical and analytical methods in rarefied gas dynamics. 8th All-Union Conference on Rarefied Gas Dynamics Proceedings. Moscow. 23-27.

5. Schavaliev, M. Sh. 1978. Burnett approximation for the distribution function and super-Burnett contribution to the tension tensor and heat flux. Appl. Math. Mech. 42(4):656-660.

6. Vasil'evsky, S. A., G. A. Tirsky, and S. V. Utjuzhnikov. 1987. Numerical method of viscous shock layer equations solution. J. Comp. Math. Phys. 27(5):741-750.

7. Chrusciel, G. T., and L. A. Pool. 1983. Knudsen layer characteristics for a highly cooled blunt body in hypersonic rarefied flow. AIAA Paper No. 1983-1424. 9 p.

8. Parker, J. G. 1959. Rotational and vibrational relaxation in diatomic gases. Phys. Fluids 2(4):449-462.

9. Chrusciel, G. T., C.H. Lewis, and T. Sugimura. 1981. Slip effects in hypersonic rarefied flow. Rarefied gas dynamics. Ed. S. S. Fisher. Progress in astronautics and aeronautics ser. 74(2):1040-1054. 